\title{
Pooling data from different populations: should there be regional differences in cerebral haemodynamics?
}

\author{
Angela S. M. Salinet ${ }^{1,2,3^{*}}$ D, Ronney B. Panerai, ${ }^{4,5}$, Juliana Caldas ${ }^{1,6}$, Ricardo C. Nogueira ${ }^{1}$, Adriana B. Conforto ${ }^{1}$, \\ Manoel J. Texeira ${ }^{1}$, Edson Bor-Seng-Shu ${ }^{1}$ and Thompson G. Robinson ${ }^{4,5}$
}

\begin{abstract}
Background: Though genetic and environmental determinants of systemic haemodynamic have been reported, surprisingly little is known about their influences on cerebral haemodynamics. We assessed the potential geographical effect on cerebral haemodynamics by comparing the individual differences in cerebral blood flow velocity (CBFv), vasomotor tone (critical closing pressure- $\mathrm{CrCP}$ ), vascular bed resistance (resistance-area product- RAP) and cerebral autoregulation (CA) mechanism on healthy subjects and acute ischaemic stroke (AIS) patients from two countries.

Methods: Participants were pooled from databases in Leicester, United Kingdom (LEI) and São Paulo, Brazil (SP) research centres. Stroke patients admitted within $48 \mathrm{~h}$ of ischaemic stroke onset, as well as age- and sex-matched controls were enrolled. Beat-to-beat blood pressure (BP) and bilateral mean CBFv were recorded during 5 min baseline. $\mathrm{CrCP}$ and RAP were calculated. CA was quantified using transfer function analysis (TFA) of spontaneous oscillations in arterial BP and mean CBFv, and the derived autoregulatory index (ARI).

Results: A total of 100 participants (50 LEl and 50 SP) were recruited. No geographical differences were found. Both LEI and SP AIS participants showed lower values of CA compared to controls. Moreover, the affected hemisphere presented lower resting CBFv and higher RAP compared to the unaffected hemisphere in both populations.

Conclusions: Impairments of cerebral haemodynamics, demonstrated by several key parameters, was observed following AIS compared to controls irrespective of geographical region. These initial results should encourage further research on cerebral haemodynamic research with larger cohorts combining different populations.
\end{abstract}

Keywords: Cerebral haemodynamics, Acute stroke, Ischaemic stroke, Cerebral autoregulation, Transcranial Doppler ultrasound

\section{Background}

Cerebral haemodynamic abnormalities may be useful early clinical markers in the development of age-related neurodegenerative disorders with vascular aetiologies [1], in determining an increased risk of stroke among healthy and diseased populations [2], and in predicting stroke prognosis [3, 4]. Due to its clinical relevance, in recent years, polling cerebral haemodynamic data from different international research centres has become, an

\footnotetext{
*Correspondence: salinetangela@gmail.com

${ }^{1}$ Neurology Department, School of Medicine, University of São Paulo, São Paulo, SP, Brazil

${ }^{2}$ Biomedical Engineering, Engineering, Modelling and Applied Social Sciences Centre, Federal ABC University, Sao Bernardo do Campo, Sao Paulo, Brazil Full list of author information is available at the end of the article
}

attractive option to generate larger study cohorts, enhancing the statistical power and the generalizability of study results $[5,6]$. Understanding to what extent regional differences affect cerebrovascular function is a key to planning international collaborative research and also to improve understanding of regional differences in treatment effectiveness and worldwide patient outcomes.

Health has multi-factorial determinants, including social, cultural, economic and environmental factors, genetic variation, and the quality of healthcare available [5]. Different continents have different health conditions, especially considering marked socioeconomic, ethnical and health care disparities. Moreover, recent studies have reported significant differences between continents in the

(C) The Author(s). 2018 Open Access This article is distributed under the terms of the Creative Commons Attribution 4.0 International License (http://creativecommons.org/licenses/by/4.0/), which permits unrestricted use, distribution, and 
prevalence of cardiovascular risk factors, such as diabetes mellitus [7, 8], hypertension [9], smoking [10] and hyperlipidaemia [11]. Nevertheless, little information on the possible international regional differences in cerebral haemodynamics is available.

Therefore, with the aim of pooling individual subjects data from different regions in an on-going international collaborative research study of cerebral haemodynamic changes following acute ischaemic stroke (AIS), we investigated systemic and cerebral haemodynamic parameters in healthy older and AIS populations derived from two different regional research centre databases in the United Kingdom and Brazil. Data from these two regions were chosen in order to ensure the homogeneity of the measurement settings and reduce the interference of other confounding factors (e.g. transcranial Doppler inter-operator reliability), as only one researcher (AS) collected and analysed the data.

\section{Methods \\ Participants}

Participants recruited by the same researcher (AS) using a standardized data collection and analysis protocol were included; participants from Leicester, United Kingdom (LEI) $[12,13]$ and Sao Paulo, Brazil (SP) were investigated. Stroke participants were consecutively recruited either from the Hyperacute Stroke Unit at the University Hospitals of Leicester NHS Trust (LEI) or from the emergency room at the Hospital das Clinicas of São Paulo (SP). Inclusion criteria were acute anterior circulation ischaemic stroke ( $<48 \mathrm{~h}$ from stroke onset) confirmed by a single acute ischaemic brain lesion on magnetic resonance image (MRI) and/or computerized tomography (CT). Exclusion criteria comprised: (1) presence of intracranial or subarachnoid haemorrhage on CT or MRI; (2) significant ipsilateral carotid artery disease (> 50\% stenosis); (3) absence of sufficient bilateral temporal bone window for insonation of the middle cerebral artery (MCA); (4) history of myocardial infarction within 6 months or other neurological disorder. Neurological impairment was assessed with the NIH Stroke Scale (NIHSS) on patient admission [14]. Age- and sex-matched healthy control subjects with no history of stroke or transient ischaemic attack, diabetes mellitus, hypercholesterolemia or carotid artery stenosis were also recruited from departmental staff and their relatives. All participants provided written informed consent in accordance with the Declaration of Helsinki; the Nottingham Research Ethics Committee 1 (LEI) and Ethics Committee of the Hospital das Clinicas (SP) approved the studies.

\section{Research protocol}

As previously described [12, 13], measurements were performed with subjects in the supine position with slight elevation of the upper body. CBF velocity (CBFv) was measured in both MCAs by insonation through the temporal bone window with $2-\mathrm{MHz}$ transducers attached to a head frame using a transcranial Doppler ultrasound (TCD) (Viasys Companion III; Viasys Healthcare and Doppler box, DWL for LEI and SP databases, respectively). Continuous non-invasive beat-to-beat blood pressure (BP) recording was performed with arterial volume clamping of the digital artery (Ohmeda 2300; Finapres, Louisville, CO, USA) with the subject's hand (left for control and unaffected hand-side for stroke group) positioned at heart level. End-tidal $\mathrm{CO}_{2}\left(\mathrm{EtCO}_{2}\right)$ was monitored using an infrared capnograph (Capnocheck Plus and MX-200 and Transmai for LEI and SP database, respectively) during nasal expiration. After stable values had been established, the servomechanism of the Finapres device was turned off and a data segment of 5 min baseline was recorded to assess systemic and cerebral haemodynamic parameters.

Data were simultaneously recorded onto a data acquisition system (PHYSIDAS, Department of Medical Physics, University Hospitals of Leicester and Doppler Box, DWL for LEI and SP databases, respectively) for subsequent off-line analysis.

\section{Data analysis}

All signals were visually inspected and narrow artefacts $(<100 \mathrm{~ms})$ were removed by linear interpolation. CBFv channels were subjected to a median filter and all signals were low-pass filtered with a cut-off frequency of $20 \mathrm{~Hz}$. The $R-R$ interval was then automatically marked from the ECG and continuous heart rate (HR) plotted against time. Mean BP and $\mathrm{CBFv}$ values were calculated for each cardiac cycle. The end of each expiratory phase was detected in the $\mathrm{EtCO}_{2}$ signal, linearly interpolated, and resampled with each cardiac cycle. The instantaneous relationship between $\mathrm{BP}$ and $\mathrm{CBFv}$ was used to estimate critical closing pressure $(\mathrm{CrCP})$ and resistance area product (RAP) for each cardiac cycle using the first harmonic method, as described in Panerai [15]. Beat-to-beat data were spline interpolated and resampled at 5 samples $\mathrm{s}^{-1}$ to produce signals with a uniform time-base.

Dynamic cerebral autoregulation (CA) represents the transient relationship between BP (stimulus or input) and CBF (response or output), and was calculated by transfer function analysis (TFA), using the parameters gain, phase and coherence, and autoregulatory index (ARI), as previously described [16, 17]. Gain and phase reflect the relative amplitude and temporal relationship between the changes in BP and CBFv over a specified frequency range, respectively. Low gain indicates intact $\mathrm{CA}$, whereas low phase (oscillations of $\mathrm{CBFv}$ and $\mathrm{BP}$ are synchronous) impaired CA. Coherence represents the fraction of output power that can be linearly explained 
by input power. Similarly to a correlation coefficient, it ranges from zero to 1 . In the absence of a linear relationship, low signal-to-noise ratio, or other variables contributing to output changes, coherence will approach zero and will reduce the reliability of gain and phase estimates. These parameters were then averaged for the very-low frequency (VLF, $0.02-0.10 \mathrm{~Hz}$ ), low frequency (LF, $0.10-0.25 \mathrm{~Hz}$ ) and high frequency $(0.25-0.40 \mathrm{~Hz})$ ranges. Inverse FFT was then applied to the TFA, converting data back into the time domain, to calculate the $\mathrm{CBFv}$ step response. ARI was assigned to each recording by using the best least squares fit between the CBFv step response and one of the 10 model ARI curves proposed by Tiecks et al. [18]. Values of ARI $=0$ represent absence of CA, while ARI $=9$ corresponds to best-observed autoregulation. ARI was calculated for each participant for both hemispheres at baseline.

\section{Statistical analysis}

Data were analysed using commercially available statistical software (SPSS 18.0, Chicago, IL). Shapiro-Wilk test was adopted to identify parameters that did not fit a normal distribution, which were then log transformed and tested again for normality. Inter-group differences were tested using $x^{2}$ test for categorical data and Student's t-test for continuous variables. Within the stroke and control groups, intra-individual differences in $\mathrm{CBFv}$, coherence, gain, phase and ARI, between LEI and SP centres were tested using two-way ANOVA with Tukey's post hoc test comparisons performed when appropriate. In addition, Levene's test was used to assess the equality of variances between the populations' variances. Levels of $p<0.05$ were considered statistically significant. The minimum sample size in each group was calculated by the formula described by Brodie et al. [19]: 44.6/ $\Delta \mathrm{ARI}^{2}$ (where $\triangle \mathrm{ARI}$ is the expected change in ARI) with $80 \%$ power at $\alpha=0.05$.

\section{Results}

A total of 100 participants were recruited from both centres, corresponding to 25 AIS patients and 25 ageand sex-matched control subjects from each centre. Baseline demographic and clinical data are described in Tables 1 and 2, respectively. No significant differences were seen in baseline demographic data, though African ethnicity, dyslipidaemia, cardiomyopathy and atrial fibrillation were more frequent in SP, and sub-surgical carotid artery stenosis $(<50 \%)$ more frequent in the LEI stroke population (Table 1). Stroke patients received medication according to local guidelines for secondary prevention at the time of assessment, with the most common antihypertensive treatment including: beta-blockers (LEI $=14(56 \%) / \mathrm{SP}=13(52 \%))$, angiotensin converting enzyme inhibitors $(\mathrm{LEI}=11(44 \%) / \mathrm{SP}=12(48 \%))$, diuretics

Table 1 Baseline characteristics of the stroke and control participants by geographical population

\begin{tabular}{|c|c|c|c|c|}
\hline \multirow[t]{2}{*}{ Variables } & \multicolumn{2}{|l|}{ Stroke } & \multicolumn{2}{|l|}{ Controls } \\
\hline & LEI $(n=25)$ & $\mathrm{SP}(n=25)$ & LEI $(n=25)$ & $\mathrm{SP}(n=25)$ \\
\hline Age, years & $60.6(15.8)$ & $62.4(11.8)$ & $61.1(9.9)$ & $60.7(10.0)$ \\
\hline aNIHSS & $11.5(6.6)$ & $14.3(5.9)$ & NA & NA \\
\hline \multicolumn{5}{|l|}{ Sex, n (\%) } \\
\hline Female & $10(40)$ & $12(48)$ & $10(40)$ & $11(44)$ \\
\hline Male & $15(60)$ & $13(52)$ & $15(60)$ & $14(56)$ \\
\hline \multicolumn{5}{|l|}{ Ethnicity, n (\%) } \\
\hline Caucasian & $20(80)$ & $15(60)$ & $23(92)$ & $22(88)$ \\
\hline African & $4(16)$ & $9(36)^{*}$ & $2(8)$ & $3(12)$ \\
\hline Natives & 0 & $1(4)$ & 0 & 0 \\
\hline Asian & $1(4)$ & 0 & 0 & 0 \\
\hline Diabetes, n (\%) & $2(8)$ & $4(16)$ & 0 & 0 \\
\hline Hypertension, n (\%) & $6(24)$ & $10(40)$ & $2(8)$ & 0 \\
\hline Dyslipidemia, n (\%) & 0 & $4(16)^{*}$ & 0 & 0 \\
\hline ICA stenosis, n (\%) & $4(16)^{*}$ & 0 & 0 & 0 \\
\hline HIV, n (\%) & $1(4)$ & 0 & 0 & 0 \\
\hline$A F, n(\%)$ & 0 & $6(24)^{*}$ & 0 & 0 \\
\hline Cardiomyopathy, n (\%) & 0 & $3(12)^{*}$ & 0 & 0 \\
\hline
\end{tabular}

Data are mean (standard deviation) or $\mathrm{n}(\%)$

LEI Leicester, UK SP, Sao Paulo, Brazil, aNIHSS admission National Institutes of Health Stroke Scale, ICA internal carotid artery, HIV human immunodeficiency virus, $A F$ atrial fibrillation

${ }^{*} p<0.02$ for comparisons between LEl and SP 
Table 2 Stroke characteristics by region

\begin{tabular}{lll}
\hline & LEI $(n=25)$ & SP $(n=25)$ \\
\hline $\begin{array}{l}\text { Stroke Side, } \mathrm{n}(\%) \\
\text { Left }\end{array}$ & $11(44)$ & $12(48)$ \\
Right & $14(56)$ & $13(52)$ \\
Stroke aetiology, n (\%) & & $18(72)$ \\
CE & $14(56)$ & 0 \\
LAA & $3(12)$ & 0 \\
SVD & $7(28)^{*}$ & 0 \\
SOE & 0 & $7(28)^{*}$ \\
SUE & $1(4)$ & $15(60)$ \\
Stroke type, n (\%) & & $10(40)^{*}$ \\
PACS & $14(56)$ & 0 \\
TACS & $4(16)$ & $9(36)$ \\
LACS & $7(28)^{*}$ & $5(20)$
\end{tabular}

Data are presented as $\mathrm{n}(\%)$

LEI Leicester, UK SP, São Paulo, Brazil, CE cardiac embolism, LAA large-artery atherosclerosis, SVD small vessel disease, SOE stroke of other determined aetiology, SUE stroke of undetermined aetiology, PACS partial anterior circulation stroke, TACS total anterior circulation stroke, LACS lacunar circulation stroke

${ }^{*} p<0.02$ for comparisons between LEI and SP

$(\mathrm{LEI}=10(40 \%) / \mathrm{SP}=7(28 \%))$. Statin therapy was prescribed to three LEI and two SP patients.

Compared to LEI stroke patients, SP patients were more likely to have total anterior circulation stroke syndrome and stroke of undetermined aetiology, and less likely to have had a lacunar stroke and small-vessel disease aetiology, though there were no differences in thrombolysis rates (Table 2). No differences between time of stroke onset and haemodynamics assessment was found ( $\mathrm{LEI}=42.0(5.9)$ and $\mathrm{SP}=39.3(7.6)$ hours).

After confirming a lack of significant inter-hemispheral difference, cerebral haemodynamic parameters $(\mathrm{CBFv}$, $\mathrm{CrCP}, \mathrm{RAP}, \mathrm{ARI}$, gain, phase and coherence) from the right and left hemispheres in control subjects were merged, as performed previously [13]. Table 3 gives the results of Levene's tests for equality of variances between LEI and SP populations. The results indicate that the population variances across regions are equivalent.

Compared to control subjects, stroke patients had significantly higher mean arterial BP, and significantly reduced $\mathrm{CBFv}$ in the affected, but not unaffected, hemisphere at the time of assessment (Table 4). RAP was significantly higher in the affected hemisphere than both the unaffected hemisphere in stroke patients, and control subjects (Table 4). No significant geographical differences were seen in either systemic or cerebral haemodynamic parameters for both stroke patients and control subjects (Table 4).

With respect to CA parameters, compared to control subjects, coherence was significantly increased in both the affected and unaffected hemispheres of AIS patients for both LF and HF ranges (Table 5). In addition, both phase in the VLF range and ARI were significantly reduced in the affected and unaffected hemispheres of AIS compared to control subjects (Table 5). No significant regional differences were observed, except for gain in the HF range between the affected and unaffected hemisphere in SP patients, which was not found in LEI patients (Table 5). Univariate ANCOVA adjusted for stroke aetiology and subtypes of the cerebral haemodynamics parameters also did not revealed any differences between regions (Table 6).

Table 3 Levene's test (statistics based on means) of variances equality between LEI and SP (geographical region) and stroke and control participants (participants type)

\begin{tabular}{|c|c|c|c|c|}
\hline & \multicolumn{2}{|c|}{ Geographical Region } & \multicolumn{2}{|c|}{ Participants Type } \\
\hline & $\bar{F}$ & $p$ value & $\bar{F}$ & $p$ value \\
\hline $\mathrm{CBFv}, \mathrm{cm} \cdot \mathrm{s}^{-1}$ & 0.721 & 0.399 & 0.195 & 0.662 \\
\hline $\mathrm{CrCP}, \mathrm{mm} \mathrm{Hg}$ & 4.276 & 0.080 & 3.153 & 0.091 \\
\hline RAP, $\mathrm{mmHg} . \mathrm{s} . \mathrm{cm}^{-1}$ & 1.496 & 0.230 & 2.231 & 0.120 \\
\hline Coherence VLF range & 2.099 & 0.493 & 2.443 & 0.407 \\
\hline Coherence LF range & 1.727 & 0.610 & 1.987 & 0.540 \\
\hline Coherence HF range & 0.929 & 0.703 & 1.592 & 0.622 \\
\hline Normalized gain VLF range, $\% \mathrm{~mm} \mathrm{Hg}^{-1}$ & 0.331 & 0.901 & 0.486 & 0.838 \\
\hline Normalized gain LF range, $\% \mathrm{~mm} \mathrm{Hg}^{-1}$ & 0.297 & 0.882 & 3.089 & 0.567 \\
\hline Normalized gain $\mathrm{HF}$ range, $\% \mathrm{~mm} \mathrm{Hg}^{-1}$ & 3.921 & 0.098 & 3.444 & 0.612 \\
\hline Phase VLF range, radians & 2.982 & 0.801 & 2.491 & 0.475 \\
\hline Phase LF range, radians & 2.001 & 0.712 & 1.984 & 0.399 \\
\hline Phase $\mathrm{HF}$ range, radians & 2.665 & 0.723 & 2.091 & 0.523 \\
\hline ARI & 0.078 & 0.762 & 0.510 & 0.950 \\
\hline
\end{tabular}


Table 4 Systemic and cerebral hemodynamic parameters in stroke and control participants by region

\begin{tabular}{|c|c|c|c|c|c|c|}
\hline \multirow[t]{3}{*}{ Variables } & \multicolumn{4}{|c|}{ Stroke Patients } & \multicolumn{2}{|l|}{ Controls } \\
\hline & \multicolumn{2}{|l|}{$\mathrm{SP}(n=25)$} & \multicolumn{2}{|l|}{ LEI $(n=25)$} & \multirow[t]{2}{*}{$\mathrm{SP}(n=25)$} & \multirow[t]{2}{*}{ LEI $(n=25$} \\
\hline & $\overline{\mathrm{AH}}$ & $\mathrm{UH}$ & $\overline{\mathrm{AH}}$ & $\mathrm{UH}$ & & \\
\hline$\overline{M A P}, \mathrm{mmHg}$ & $109.2(5.7)^{\#}$ & & $103.0(5.5)^{\#}$ & & $90.0(6.1)$ & $89.2(3.9)$ \\
\hline $\mathrm{CBFv}, \mathrm{cm}^{-\mathrm{s}^{-1}}$ & $47.5(6.3)^{\#}$ & $55.1(5.8)$ & $41.7(2.4)^{*}$ & $45.03(2.8)$ & $60.3(11.6)$ & $53.8(5.5)$ \\
\hline $\mathrm{CrCP}, \mathrm{mm} \mathrm{Hg}$ & $18.2(6.7)$ & $18.0(4.6)$ & $19.4(5.0)$ & $17.0(5.5)$ & $14.2(4.47)$ & $15.5(8.0)$ \\
\hline RAP, $\mathrm{mmHg} . \mathrm{s} . \mathrm{cm}^{-1}$ & $1.97(0.32)^{* \#}$ & $1.76(0.25)^{\#}$ & $2.25(0.23)^{* \#}$ & $1.77(0.17)^{\#}$ & $1.56(0.27)$ & $1.65(0.60)$ \\
\hline
\end{tabular}

Data are presented as mean (SD)

LEI Leicester, UK SP, Sao Paulo, Brazil, AH affected hemisphere, UH unaffected hemisphere, MAP Mean arterial pressure, CBFv Cerebral blood flow velocity, CrCP Critical closing pressure, RAP Resistance Area Product

${ }^{*} p<0.01$, Tukey's post-hoc test for comparisons between stroke affected and unaffected hemispheres

$\# p<0.05$, Tukey's post-hoc test for comparisons between controls and stroke participants (same geographical region)

\section{Discussion}

\section{Main findings}

To the best of our knowledge, this is the first study to compare CA in participants from different geographical regions with marked environmental and socio-economic differences. These pooled analyses suggest no geographical differences in key, commonly measured cerebral haemodynamic parameters, including $\mathrm{CBFv}, \mathrm{CrCP}$, RAP and ARI. Overall, and in keeping with previous studies, impairment of cerebral haemodynamic parameters was reported in stroke compared to control participants, particularly in the affected hemisphere. Importantly, no geographical regional differences were found.

\section{Clinical relevance}

Recognizing similarities and differences in the cerebral haemodynamics in healthy subjects and stroke has public health and clinical implications for several cardiovascular outcomes. Haemodynamic abnormalities may be useful early clinical markers in the development of age-related vascular neurodegenerative disorders. Describing cerebral haemodynamic changes and their regulation in different geographical populations may help understand differences across age and ethnicities, as well as the potential generalizability of clinical studies results.

Impairment of $\mathrm{CBFv}$ and $\mathrm{CA}$ has a direct and major impact on secondary brain injury and clinical outcomes [3, 4], as well as on planning effective therapeutic strategies that consider BP management and early mobilization protocols. In line with previous studies, our results showed a deterioration of important haemodynamic parameters in the acute phase of ischaemic stroke, particularly reduced phase and ARI values compared to controls [3, 20-23]. Moreover, no significant alteration in gain between groups was found $[6,20]$. Though TFA became a popular approach for assessment of dynamic CA, very few acute stroke studies

Table 5 Dynamic CA parameters in stroke and control participants by geographical region

\begin{tabular}{|c|c|c|c|c|c|c|}
\hline \multirow[t]{3}{*}{ Variables } & \multicolumn{4}{|c|}{ Stroke Patients } & \multicolumn{2}{|l|}{ Controls } \\
\hline & \multicolumn{2}{|l|}{$\mathrm{SP}(n=25)$} & \multicolumn{2}{|l|}{ LEI $(n=25)$} & \multirow[t]{2}{*}{$\mathrm{SP}(n=25)$} & \multirow[t]{2}{*}{ LEI $(n=25)$} \\
\hline & $\overline{\mathrm{AH}}$ & $\mathrm{UH}$ & $\overline{\mathrm{AH}}$ & $\mathrm{UH}$ & & \\
\hline Coherence VLF range & $0.65(0.19)$ & $0.64(0.14)$ & $0.61(0.14)$ & $0.59(0.14)$ & $0.52(0.16)$ & $0.48(0.11)$ \\
\hline Coherence LF range & $0.68(0.23)^{\#}$ & $0.73(0.19)^{\#}$ & $0.68(0.17)^{\#}$ & $0.71(0.15)^{\#}$ & $0.56(0.18)$ & $0.58(0.15)$ \\
\hline Coherence HF range & $0.72(0.18)^{\#}$ & $0.77(0.10)^{\#}$ & $0.69(0.17)^{\#}$ & $0.67(0.16)^{\#}$ & $0.52(0.21)$ & $0.51(0.15)$ \\
\hline Normalized gain VLF range, $\% \mathrm{~mm} \mathrm{Hg}^{-1}$ & $1.26(0.57)$ & $1.17(0.66)$ & $0.94(0.33)$ & $0.91(0.34)$ & $1.01(0.39)$ & $1.19(0.43)$ \\
\hline Normalized gain LF range, $\% \mathrm{~mm} \mathrm{Hg}^{-1}$ & $1.32(0.48)$ & $1.22(0.46)$ & $1.35(0.44)$ & $1.26(0.69)$ & $1.45(0.83)$ & $1.49(0.78)$ \\
\hline Normalized gain $\mathrm{HF}$ range, $\% \mathrm{~mm} \mathrm{Hg}{ }^{-1}$ & $1.50(0.74)^{*}$ & $1.36(0.51)$ & $1.36(0.54)$ & $1.28(0.55)$ & $1.35(0.63)$ & $1.40(0.58)$ \\
\hline Phase VLF range, radians & $0.53(0.42)^{\#}$ & $0.78(0.39)^{\#}$ & $0.58(0.50)^{\#}$ & $0.70(0.49)^{\#}$ & $0.88(0.35)$ & $0.87(0.41)$ \\
\hline Phase LF range, radians & $0.30(0.45)$ & $0.25(0.38)$ & $0.30(0.35)$ & $0.31(0.39)$ & $0.48(0.19)$ & $0.45(0.41)$ \\
\hline Phase HF range, radians & $0.09(0.29)$ & $0.10(0.25)$ & $0.11(0.40)^{\#}$ & $0.17(0.21)^{\#}$ & $0.05(0.21)$ & $0.01(0.28)$ \\
\hline ARI & $4.8(2.3)^{\#}$ & $4.9(2.0)^{\#}$ & $5.1(1.8)^{\#}$ & $5.0(1.1)^{\#}$ & $5.9(1.5)$ & $5.5(1.2)$ \\
\hline
\end{tabular}

Data are presented as mean (SD)

LEI Leicester, UK SP, Sao Paulo, Brazil, AH affected hemisphere, UH unaffected hemisphere, ARI Autoregulatory Index, VLF, LF, HF Very low, low and high frequency ranges, respectively

${ }^{*} p<0.01$, Tukey's post-hoc test for comparisons between stroke affected and unaffected hemispheres

$\# p<0.05$, Tukey's post-hoc test for comparisons between controls and stroke participants 
Table 6 ANCOVA and ANOVA results for comparison of cerebral haemodynamics parameters between geographical regions (stroke only)

\begin{tabular}{|c|c|c|c|c|c|c|}
\hline & \multicolumn{2}{|l|}{ MS } & \multicolumn{2}{|l|}{ F-statistic } & \multicolumn{2}{|l|}{$p$-Value } \\
\hline & ANCOVA & $\overline{\text { ANOVA }}$ & ANCOVA & ANOVA & ANCOVA & $\overline{\text { ANOVA }}$ \\
\hline $\mathrm{CBFv}, \mathrm{cm}_{\mathrm{s}}^{-1}$ & 3.2 & 8.6 & 0.7 & 0.9 & 0.83 & 0.42 \\
\hline $\mathrm{CrCP}, \mathrm{mm} \mathrm{Hg}$ & 2.5 & 3.0 & 5.2 & 4.8 & 0.60 & 0.81 \\
\hline RAP, mmHg.s.cm ${ }^{-1}$ & 4.9 & 3.0 & 6.1 & 1.8 & 0.10 & 0.24 \\
\hline Coherence VLF range & 5.7 & 9.2 & 3.2 & 4.5 & 0.31 & 0.19 \\
\hline Coherence LF range & 3.8 & 4.4 & 2.9 & 4.3 & 0.44 & 0.31 \\
\hline Coherence HF range & 3.1 & 5.3 & 2.3 & 3.6 & 0.56 & 0.29 \\
\hline Normalized gain VLF range, $\% \mathrm{~mm} \mathrm{Hg}^{-1}$ & 14.0 & 5.1 & 5.2 & 1.4 & 0.07 & 0.33 \\
\hline Normalized gain LF range, $\% \mathrm{~mm} \mathrm{Hg}^{-1}$ & 8.1 & 4.5 & 0.9 & 2.3 & 0.30 & 0.12 \\
\hline Normalized gain $\mathrm{HF}$ range, $\% \mathrm{~mm} \mathrm{Hg}^{-1}$ & 7.8 & 4.4 & 5.5 & 1.2 & 0.21 & 0.40 \\
\hline Phase VLF range, radians & 4.2 & 2.5 & 0.2 & 0.1 & 0.90 & 0.90 \\
\hline Phase LF range, radians & 6.7 & 1.8 & 0.2 & 0.4 & 0.80 & 0.90 \\
\hline Phase HF range, radians & 5.5 & 1.4 & 1.0 & 0.2 & 0.80 & 0.30 \\
\hline ARI & 1.5 & 1.9 & 4.5 & 3.9 & 0.51 & 0.77 \\
\hline
\end{tabular}

The analysis of covariance (ANCOVA) model included stroke aetiology and subtypes as covariates

ANOVA analysis of variance, $M S$ mean square, $A H$ affected hemisphere, $U H$ unaffected hemisphere, $V L F, L F, H F$ Very low, low and high frequency ranges, respectively

$p$-value for ANOVA (geographical interaction) and ANCOVA controlled for stroke aetiology and subtype

have reported the use of coherence estimates [3, 22]. These previous studies failed to find raised coherence values in acute stroke, but an analogous parameter (the squared correlation coefficient) was found to be increased bilaterally after the first $48 \mathrm{~h}$ of stroke onset, suggesting worsening of CA [23].

The importance and novelty of our results, however, stem from the similarities of the haemodynamic responses from research centres in both UK and Brazil, irrespective of the participant group (stroke or healthy controls). Despite the potential differences in dietary habits and lifestyles, and particularly in SP participants, higher prevalence of uncontrolled hypertension and widespread use of over-the-counter medications, cerebral haemodynamic parameters were not significantly different in our study. The authors believe that the outcome of this paper will strengthen the argument in favour of multicentre and multinational collaborative studies of the impact of cerebral haemodynamics in stroke and other conditions, such as sepsis and traumatic brain injury.

\section{Environment effects on cerebral haemodynamics}

Since differences in CBF and its control mechanisms between European and South American populations have not been previously reported, it is difficult to assess consistency with other studies in the literature. A previous TCD study of healthy young participants $(n=20)$ in Germany and Hong Kong described no CBFv differences in the posterior cerebral arteries at rest and during cerebral activation [24]. Nevertheless, slower initial haemodynamic responses to visual activation paradigms were described in the Asian group that may be related to deficits in the nitric oxide system. Previous studies have also compared cerebral haemodynamic responses between South Asian and Caucasian participants, but the results were inconsistent. In a United Kingdom-based study, resting MCA CBFv and cerebrovascular resistance parameters (Pourcelot's resistive index and Gosling's pulsatility index) were significantly higher, and CA impaired in South Asian participants [25]. By contrast, no difference in the same parameters of CBFv and cerebrovascular resistance was found in another recent study of Canadian-based South Asians and Caucasians [26].

Disparities in cardiovascular and cerebrovascular health and mortality among populations have been well documented, but poorly understood [27, 28]. Studies have shown that the prevalence and mortality from hypertensive heart disease, stroke, and renal disease are higher among individuals of African compared to Caucasian descent [29]. Moreover, intracranial atherosclerosis is highly prevalent among patients with Asian, Hispanic, and African ancestry [30].

The present study did not demonstrate significant geographical differences in cerebral haemodynamic parameters between UK and Brazilian populations, with exception of gain at high frequency band. Immink and colleagues (2005) found an increase in gain only at higher frequencies in MCA-only stroke group. This may be the reason for the difference between regions, as SP comprised MCA infarcts only, whereas seven lacunar strokes were included in the LEI group (Table 2). Though recommended by the Cerebral Autoregulation Network (CARNet) [31], there is 
limited information in the literature on CA status in the complete frequency dependence of coherence, gain and phase in the range $0.02-0.40 \mathrm{~Hz}$. While the TFA parameters changes in VLF and LF ranges are familiar, very little is known about the behaviour of the BP/CBFv system at frequencies higher than $0.25 \mathrm{~Hz}$. In the TFA model, phase and gain are considered two different aspects of a high-pass filter that acts primarily in the VLF and LF ranges. At high frequencies, $\mathrm{CA}$ is considered less relevant and CBFv changes are associated with heart stroke volume beat-to-beat variability. More clinical studies are necessary to investigate the clinical importance of investigating $\mathrm{CA}$ in the higher frequency band, taking advantage of the work that has already been done. A further finding of the present study related to the responses of cerebrovascular resistance mechanisms, derived from a two-parameter model: RAP-CrCP. Levene's test revealed a marginal difference in $\mathrm{CrCP}$ between geographical regions, with a tendency to higher values in LEI participants. Further research is needed to assess the clinical value of this finding.

\section{Standardized protocol}

The experimental design of previous cerebral haemodynamic studies has been inconsistent, making it difficult to compare results directly [32, 33]. Furthermore, there have been no previous CA studies in either older or stroke populations in Brazil. In contrast, in the UK, some studies have previously described the haemodynamic responses to spontaneous BP fluctuations, carbon dioxide modulations and brain activation paradigms in both populations [12, 13, 34, 35]. More recently, the Department of Cardiovascular Sciences at the University of Leicester (Leicester, UK) has constructed a large database incorporating recordings from a series of separate studies performed in the same laboratory, using similar protocols, operator training and equipment [36]. They have presented normative values for cerebral haemodynamic parameters in a large healthy population indicating parameters that may help distinguish between normal and abnormal CA.

The present study was only possible due to the development of standardized data collection and analysis that provided a robust approach for the systematic evaluation of $\mathrm{CBFv}$ and its main regulatory mechanisms [37]. Similar to the Leicester normative study [36], all data collected in this study were acquired with similar study protocols and laboratory set-up, albeit with minor differences in the equipment used, and most importantly, without observer variability since all recordings were performed by only one researcher (AS). This avoids inter-observer variability in the study protocol (particularly concerning the TCD data), and it consequently increases the reproducibility of study reports.

\section{Study limitations}

This study has limitations, including the use of non-invasive measurements of $\mathrm{CBF}$ and $\mathrm{BP}$. Another limitation to consider is stroke patients received medication according to local guidelines for secondary prevention at the time of assessment. Though patients were on vasoactive therapy at time of assessment, no significant difference between populations was found. Although TFA and the ARI index can be regarded as the most widely used approach for assessment of dynamic CA, it is important to note that neither can be regarded as a 'gold standard'. Future studies are needed to replicate our findings using alternative approaches such as time-domain analysis or the Mx index [12]. Finally, the sample size is relatively small, and is unlikely to be representative enough to ensure robust conclusions. Therefore, the authors strongly advocate a large multicentre validation study with larger sample size to explore further the possibility of regional geographical influences on cerebral haemodynamics, and possible mechanisms to support any differences.

\section{Conclusion}

In conclusion, our results showed no significant differences in selected cerebral haemodynamic parameters between two different socio-economical geographical regions. In both research centres, acute ischaemic stroke depressed key measures of CA compared to a healthy older control population. These findings encourage further larger international studies of cerebral haemodynamic changes following AIS by pooling individual subject's data from different regions.

\section{Abbreviations}

AIS: Acute ischaemic stroke; ARI: Autoregulatory index; BP: Blood pressure; CA: Cerebral autoregulation; CBF: Cerebral blood flow; CBFv: Cerebral blood flow velocity; CrCP: Critical closing pressure; CT: Computerized tomography; $\mathrm{EtCO}_{2}$ : End-tidal $\mathrm{CO}_{2}$; HR: Heart rate; LEl: Leicester; MCA: Middle cerebral artery; MRI: Magnetic resonance image; NHISS: NIH Stroke Scale; RAP: Resistance area product; SP: Sao Paulo; TCD: Transcranial doppler ultrasound; TFA: Transfer function analysis

\section{Acknowledgements}

Authors acknowledge Dr. João Loures Salinet Junior for his contribution in the data analysis and revising the manuscript. TGR is an NIHR Senior Investigator.

\section{Funding}

The work was supported by the Brazilian Ministry of Education (Grant number 0411-10-8) and São Paulo Research Foundation (Grant number 2014/04955-8).

\section{Availability of data and materials}

The datasets used and/or analysed during the current study are available from the corresponding author on reasonable request.

\section{Authors' contributions}

ASMS, RBP and TGR made a substantial contribution to the concept and design; ASMS, JC, RBP made the acquisition of data or analysis and interpretation of data; ASMS, TGR, RBP drafted the article; JC, RBP, RCN, TGR, $A B C, M J T, E B S S$ revised it critically for important intellectual content; and ASMS, $J C, R B P, R C N, T G R, A B C, M J T, E B S S$ approved the final version to be published. 


\section{Ethics approval and consent to participate}

All participants provided written informed consent in accordance with the Declaration of Helsinki; the Nottingham Research Ethics Committee 1 (Ref: 11/EM/0016) and Ethics Committee of the Hospital das Clinicas (N. 982.280) approved the studies. In all consent forms, patient's relatives (wife, husband or offspring) were asked to also sign the form as witness. In case of patients doubtfully capable or incapable of consent, the next of kin (wife, husband or offspring) was asked to provide consent.

\section{Consent for publication}

N/A

\section{Competing interests}

The authors declare that they have no competing interests.

\section{Publisher's Note}

Springer Nature remains neutral with regard to jurisdictional claims in published maps and institutional affiliations.

\section{Author details \\ 'Neurology Department, School of Medicine, University of São Paulo, São Paulo, SP, Brazil. 'Biomedical Engineering, Engineering, Modelling and Applied Social Sciences Centre, Federal ABC University, Sao Bernardo do Campo, Sao Paulo, Brazil. ${ }^{3}$ Faculty of Physiotherapy, Ibirapuera University, São Paulo, Brazil. ${ }^{4}$ Department of Cardiovascular Sciences, Cerebral Haemodynamics in Ageing and Stroke Medicine Research Group, University of Leicester, Leicester, UK. ${ }^{5} \mathrm{NIHR}$ Leicester Biomedical Research Centre, University of Leicester, Leicester, UK. ${ }^{6}$ Critical Care Unit Hospital São Rafael, Salvador, Brazil.}

Received: 21 March 2018 Accepted: 14 September 2018

Published online: 27 September 2018

\section{References}

1. Keage HAD, Churches OF, Kohler M, Pomeroy D, Luppino R, Bartolo ML, Elliotta S. Cerebrovascular function in aging and dementia: a systematic review of transcranial Doppler studies. Dement Geriatr Cogn Dis Extra. 2012; 2:258-70

2. Derdeyn CP, Grubb RL Jr, Powers WJ. Cerebral hemodynamic impairment: methods of measurement and association with stroke risk. Neurology. 1999; 53:251-61.

3. Castro P, Serrador JM, Rocha I, Sorond F, Azevedo E. Efficacy of cerebral autoregulation in early ischemic stroke predicts smaller infarcts and better outcome. Front Neurol. 2017:8:113-23.

4. Reinhard M, Rutsch S, Lambeck J, Wihler C, Czosnyka M, Weiller C, et al. Dynamic cerebral autoregulation associates with infarct size and outcome after ischemic stroke. Acta Neurol Scand. 2012;125:156-62.

5. The Nuffield Council on Bioethics. The ethics of research related to healthcare in developing countries. London: Nuffield Council on Bioethics; 2002.

6. Hedman K, Alm A. A pooled data analysis of three randomised, doublemasked six-month clinical studies comparing the intraocular pressure reducing effect of latanoprost and timolol. European J Ophthalmol. 2000;2:94-104.

7. Danaei G, Finucane MM, Lu Y, et al. National, regional, and global trends in fasting plasma glucose and diabetes prevalence since 1980: systematic analysis of health examination surveys and epidemiological studies with 370 country-years and 2.7 million participants. Lancet. 2011;378:31-40.

8. Guariguata L, Whiting DR, Hambleton I, et al. Global estimates of diabetes prevalence for 2013 and projections for 2035. Diabetes Res Clin Pract. 2014; 103:137-49.

9. Danaei G, Finucane MM, Lin JK, et al. National, regional, and global trends in systolic blood pressure since 1980: systematic analysis of health examination surveys and epidemiological studies with 786 country-years and 5.4 million participants. Lancet. 2011;377:568-77.

10. Ng M, Freeman MK, Fleming TD, et al. Smoking prevalence and cigarette consumption in 187 countries, 1980-2012. JAMA. 2014;311:183-92.

11. Farzadfar F, Finucane MM, Danaei G, et al. National, regional, and global trends in serum total cholesterol since 1980: systematic analysis of health examination surveys and epidemiological studies with 321 country-years and 3.0 million participants. Lancet. 2011;377:578-86.
12. Salinet $A S M$, Robinson $T G$, Panerai RB. Effects of cerebral ischemia on human neurovascular coupling, CO2 reactivity, and dynamic cerebral autoregulation. J Appl Physiol. 2015;118:170-7.

13. Salinet ASM, Robinson TG, Panerai RB. The longitudinal evolution of cerebral blood flow regulation after acute ischaemic stroke. Cerebrovasc Dis Extra. 2014:4:186-97.

14. Lyden P, Brott T, Tilley B, Welch KMA, Mascha EJ, Levine S, et al. Improved reliability of the NIH stroke scale using video training. Stroke. 1994;25:2220-6.

15. Panerai RB. The critical closing pressure of the cerebral circulation. Med Eng Phys. 2003;25:621-32.

16. Katsogridakis E, Bush G, Fan L, Birch AA, Simpson DM, Allen R, Potter $J$ J, et al. Detection of impaired cerebral autoregulation improves by increasing arterial blood pressure variability. J Cereb Blood Flow Metab. 2013;33:519-23

17. Meel van den ASS, Simpson DM, Wang LY, Zhang R, Tarumi T, Rickards CA et al. Between Centre variability in transfer function analysis, a widely used method for linear quantification of the dynamic pressure-flow relation: the CARNet study. Med Eng Physics. 2014;36:620-7.

18. Tiecks FP, Lam AM, Aaslid R, Newell DW. Comparison of static and dynamic cerebral autoregulation measurements. Stroke. 1995;26:1014-9.

19. Brodie FG, Atkins ER, Robinson TG, Panerai RB. Reliability of dynamic cerebral autoregulation measurement using spontaneous fluctuations in blood pressure. Clin Sci. 2009;116:513-20.

20. Ma H, Guo Z-N, Liu J, Xing Y, Zhao R, Yang Y. Temporal course of dynamic cerebral autoregulation in patients with Intracerebral hemorrhage. Stroke. 2016:47:674-81

21. Eames PJ, Blake MJ, Dawson SL, Panerai R, Poter J. Dynamic cerebral autoregulation and beat to beat blood pressure control are impaired in acute ischaemic stroke. J Neurol Neurosurg Psychiatry. 2002;72:467-72.

22. Immink RV, van Montfrans GA, Stam J, Karemaker JM, Diamant M, van Lieshout JJ. Dynamic cerebral autoregulation in acute lacunar and middle cerebral artery territory ischemic stroke. Stroke. 2005:36:2595-600.

23. Reinhard M, Wihler C, Roth M, Harloff A, Niesen WD, Timmer J, Weiller C, Hetzel A. Cerebral autoregulation dynamics in acute ischemic stroke after rtPA thrombolysis. Cerebrovasc Dis. 2008;26:147-55.

24. Hao Q, Wong LK, Lin WH, Leung TW, Kaps M, Rosengarten B. Ethnic influences on neurovascular coupling: a pilot study in whites and Asians. Stroke. 2010:41:383-94.

25. Bathula R, Hughes AD, Panerai RB, Potter JF, AS MGT, Tillin T, et al. South Asians have adverse cerebrovascular haemodynamics, despite equivalent blood pressure, compared with Europeans. This is due to their greater hyperglycaemia. Int J Epidemiol. 2011;40:1490-8.

26. Booth HP, Prevost AT, Gulliford MC. Severity of obesity and management of hypertension, hypercholesterolemia and smoking in primary care: population-based cohort study. J Hum Hypetens. 2015;2:1-6.

27. Sheth T, Nair C, Nargundkar M, Anand S, Yusuf S. Cardiovascular and cancer mortality among Canadians of European, south Asian and Chinese origin from 1979 to 1993: an analysis of 1.2 million deaths. CMAJ. 1999;161:132-8.

28. Rambihar VS, Rambihar SP, Rambihar VS. Race, ethnicity, and heart disease: challenge for cardiology for the 21st century. Am Heart J. 2010;159:1-14.

29. Burt VL, Whelton P, Roccella EJ, Brown C, Cutler JA, Higgins M, et al. Prevalence of hypertension in the US adult population. Results from the third National Health and nutrition examination survey, 1988-1991. Hypertension. 1995;25:305-13.

30. Mille E, Levin J, Brendel M, Zach C, Barthel H, Sabri O. Etal. Cerebral glucose metabolism and dopaminergic function in patients with Corticobasal syndrome. J Neuroimaging. 2016:27:1-7.

31. Claassen JA, Meel-van den Abeelen AS, Simpson DM, Panerai RB, International Cerebral Autoregulation Research Network (CARNet). Transfer function analysis of dynamic cerebral autoregulation: a white paper from the International Cerebral Autoregulation Research Network. J Cereb Blood Flow Metab. 2016:36:665-80.

32. van Beek AH, Claassen JA, Rikkert MG, Jansen RW. Cerebral autoregulation: an overview of current concepts and methodology with special focus on the elderly. J Cereb Blood Flow Metab. 2008;28:1071-85.

33. Aries M, Elting JW, De Keyser J, Kremer BPH, Vroomen P. Cerebra autoregulation in stroke a review of Transcranial Doppler studies. Stroke. 2010;41:12-20.

34. Saeed NP, Panerai RB, Horsfield MA, Robinson TG. Does stroke subtype and measurement technique influence estimation of cerebral autoregulation in acute ischaemic stroke? Cerebrovasc Dis. 2013;35:257-61. 
35. Minhas JS, Syed NF, Haunton VJ, Panerai RB, Robinson TG, Mistri AK. Is dynamic cerebral autoregulation measurement using transcranial Doppler ultrasound reproducible in the presence of high concentration oxygen and carbon dioxide? Physiol Meas. 2016:37:673-82.

36. Patel P, Panerai RP, Haunton V, Katsogridakis E, Saeed NP, Salinet ASM, et al. The Leicester cerebral haemodynamics database: normative values and the influence of age and sex. Physiol Meas. 2016;37:1485-99.

37. Giller CA, Bowman G, Dyer H, Mootz L, Krippner W. Cerebral arterial diameters during changes in blood pressure and carbon dioxide during craniotomy. Neurosurgery. 1993;27:737-41

Ready to submit your research? Choose BMC and benefit from:

- fast, convenient online submission

- thorough peer review by experienced researchers in your field

- rapid publication on acceptance

- support for research data, including large and complex data types

- gold Open Access which fosters wider collaboration and increased citations

- maximum visibility for your research: over $100 \mathrm{M}$ website views per year

At BMC, research is always in progress.

Learn more biomedcentral.com/submissions 